\title{
CAPACIDADE PREDATÓRIA DE Chrysoperla externa ALIMENTADA COM Frankliniella schultzei EM PLANTAS DE ALFACE DE CULTIVO HIDROPÔNICO
}

\author{
Leonardo R. Barbosa ${ }^{1}$ \\ Alexander M. Auad ${ }^{1}$ \\ Sérgio de Freitas ${ }^{1}$
}

\section{RESUMO}

Este estudo teve como objetivo avaliar em laboratório a capacidade predatória de larvas de Chrysoperla externa (Hagen), quando alimentadas com adultos de Frankliniella schultzei (Trybom) criados em alface, produzida em cultivo hidropônico. Os experimentos foram realizados à temperatura de $25 \pm 2{ }^{\circ} \mathrm{C}$, umidade relativa de $70 \pm 10 \%$ e fotofase de 14 horas. Para as larvas de diferentes regimes alimentares foram fornecidos discos de alface de $2 \mathrm{~cm}$ de diâmetro contendo uma quantidade de tripes (adulto) suficiente para a alimentação dos predadores num intervalo de $24 \mathrm{~h}$, após o qual fazia-se a contagem do número consumidos por cada larva. Utilizou-se delineamento inteiramente casualizado com seis tratamentos e sete repetições. Os tratamentos foram definidos com base na alimentação anterior das larvas. Verificou-se um maior número de $F$. shultzei consumidos por larvas de $C$. externa de $3^{\circ}$ instar, quando comparadas com larvas de $1^{\circ}$ e $2^{\underline{o}}$ ínstares, independentemente dos regimes alimentares dessas. Larvas de $3^{\circ}$ ínstar, alimentadas nos ínstares anteriores com S. cerealella (3SST) apresentaram consumo estatisticamente maior do que larvas no mesmo ínstar de diferentes regimes alimentares (3TTT e 3STT); o mesmo não ocorreu para larvas de $2^{\circ}$ ínstar (2TT e 2ST), as quais o alimento não influenciou. Foi constatado período médio em dias

'Departamento de Fitossanidade, FCAV/ UNESP, Via de Acesso Paulo Donato Castellani, $\mathrm{s} / \mathrm{n}^{\mathrm{o}}$, 148700-000, Jaboticabal, SP, Brasil. 
de 5,$43 ; 4,00$ e 3,14 para as larvas $1 \mathrm{~T}, 2 \mathrm{TT}$ e $2 \mathrm{ST}$, respectivamente. Para larvas de $3^{\circ}$ ínstar as avaliações foram realizadas por até 15 dias. Neste intervalo, constatou-se que larvas que se alimentaram nos ínstares anteriores com tripes (3TTT) não átingiram a fase posterior (pupa), da mesma forma para larvas que começaram a se alimentar com tripes a partir do $2 \circ$ ínstar (3STT), apresentando $43 \%$ de mortalidade antes de finalizar esse período. Porém larvas de $3^{2}$ ínstar alimentadas anteriormente com $S$. cerealella (3SST) levaram em média 9,14 dias para atingir a fase posterior.

Palavras-chave: Insecta, crisopídeo, tripes, alface.

\section{ABSTRACT}

\section{PREDATORY CAPACITY OF Chrysoperla Externa (HAGEN) (NEUROPTERA: CHRYSOPIDAE) FED Frankliniella Schultzei (TRYBOM) (THYSANOPTERA: THRIPIDAE) IN HYDROPONIC GROWING LETTUCE PLANTS.}

This work had the objetive of evaluating in laboratory the predatory capacity of $C$. externa, feeded on $F$. schultzei and reared in lettuce produced in hydroponic system. The experiments were undertaken at $25 \pm 2{ }^{\circ} \mathrm{C}$ temperature, $70 \pm 10 \%$ relative humidity and $14 \mathrm{~h}$ photophase. For larval different feeding regimes, lettuce discs of $2 \mathrm{~cm}$ diameter were given containing a quantity of adult thrips enough for predators feeding in $24 \mathrm{~h}$ interval, after which the counting of consumed number of thrips by each larva was performed. The completely randomized design was used with six treatments and seven replicates. The treatments were defined in anterior larvae feeding basis. A higher number of $F$. schultzei consumed by $C$ externa larvae of $3^{\text {rd }}$ instar, compared to $1^{\text {st }}$ and $2^{\text {nd }}$ instars larval independently on their feeding regimen was observed. Larvae of $3^{\text {rd }}$ ins tar, fed Sitotrog a cerealella (3SST) in anterior instars showed a statistically higher consumption than larvae at same instar but in different feeding regime (3TTT and 3STT), for $2^{\text {nd }}$ instar larvae (2TT and 2ST) the same 
did not occur. It was observad a period of 5.43, 4.00 and 3.14 days for $1 \mathrm{~T}$, 2TT and 2ST larvae, respectively. For $3^{\text {rd }}$ instar larvae the evaluation was made during 15 days. In this interval it was verified that larvae fed with thrips (3TTT) in anterior instars did not reach the posterior phase (pupa). The same occured for larvae that sarted to be fed thrips from $2^{\text {nd }}$ instar (3STT), presenting $43 \%$ of mortality. However, $3^{\text {rd }}$ instar larvae fed before with $S$. cerealella (3SST) took the average of 9.14 days to reach the later phase.

Key words: Insecta, chrysopids, thrips, lettuce.

\section{INTRODUÇÃO}

No Brasil, a alface é produzida de diferentes modos, dentre eles, o cultivo hidropônico, que consiste na utilização de solução nutritiva como substrato para as plantas, e que vem ultimamente se destacando.

Dos insetos-pragas mais frequientes na alface, os tripes vêm assumindo papel importante, em função da diversidade adaptativa que os tem capacitado a explorar, com sucesso, diversos nichos ambientais (Lewis, 1973; Ananthakrishnan, 1993). Yudin et al. (1988) encontraram doze espécies de tripes associados à cultura da alface, sendo Frankliniella occidentalis (Pergande) a mais abundante, seguida por Frankliniella schultzei (Trybom). A alimentação desses insetos em plantas tem como conseqüência a extração do conteúdo celular, a formação de áreas descoradas e o aparecimento, nos locais atacados, de pontos ferruginosos (necrose dos tecidos) ou pardo-enegrecidos (deposição de gotas fecais). Em algumas situações, quando presente nas flores, podem ser benéficos, auxiliando a polinização, ou maléficos, quando danificam, esterilizam e impedem a formação de frutos (Lima, 1938).

Muitos problemas têm surgido no que se refere ao controle dos insetos-pragas em ambiente fechado, pois a utilização intensiva de tratamentos químicos pode aumentar o risco de fitotoxidade e o desenvolvi- 
mento de resistência (Principi, 1984). Isto evidencia a importância de técnicas alternativas nesse sistema de cultivo. Assim, para o controle dos tripes em alface, produzida em cultivo hidropônico, é promissor 0 uso de crisopídeos.

Freitas \& Fernandes (1996) ressaltaram que além da voracidade, os crisopídeos apresentam elevada plasticidade ecológica, sendo encontrados em diferentes agroecossistemas, associados a diferentes pragas. Ainda, segundo eles, esses predadores se alimentam de grande variedade de insetos sugadores de seiva (pulgões, cochonilhas e psilídios), ácaros e tripes.

Paine (1992) verificou que uma larva de terceiro ínstar de Chrysoperla carnea (Stevens) consumiu em 24 horas, 17,91 e 9,64 adultos de Gynaikothrips ficorum Marchal (Thysanoptera: Phlaeothripidae), em média, quando lhes foram oferecidos 100 e 30 indivíduos, respectivamente.

A duração dos estágios de $1^{\circ}, 2^{\circ}, 3^{\text {o }}$ ínstar e período larval total de $C$. carnea foram de 3,$57 ; 2,45 ; 7,46$ e 14,18 dias, respectivamente, quando alimentadas com Thrips tabaci Lind e de 3,10; 3,00; 3,87 e 10,48 dias, respectivamente, quando alimentadas com $G$. ficorum (Awadallah et al., 1975).

Este trabalho teve como objetivo estudar em laboratório a capacidade predatória de larvas Chrysoperla externa (Hagen) alimentadas com Feanklinella schultzei (Trybon) criadas em alface, produzida em cultivo hidropônico.

\section{MATERIAL E MÉTODOS}

Os experimentos foram realizados no Laboratório de Biossistemática e Criação Massal de Crisopídeos Crysoperla externa (Hagen) (Neuroptera, Chrysopidae) da Faculdade de Ciências Agrárias e Veterinária, Câmpus de Jaboticabal, em condições controladas de 
temperatura $\left(25 \pm 2^{\circ} \mathrm{C}\right)$, de umidade relativa $(70 \pm 10 \%)$ e em fotofase de 14 horas.

Os tripes Frankliniella schultzei (Trybom) (Thysanoptera, Thripidae) utilizados para alimentar as larvas de crisopídeos foram coletados em cultivos de alface na região de Jaboticabal. Depositados sobre folhas novas de alface em cultivo hidropônico, foram posteriormente utilizados para a realização dos testes de alimentação.

Os tratamentos foram definidos com base na alimentação anterior das larvas do predador: Larvas de $1^{2}$ ínstar foram alimentadas desde 0 inicio com tripes (1T); larvas de $2^{\circ}$ ínstar foram alimentadas anteriormente com tripes (2TT) ou com $S$. cerealella (2ST); larvas de 3 o ínstar foram alimentadas desde o inicio com tripes (3TTT), alimentadas $\operatorname{com} S$. cerealella no primeiro ínstar e tripes a partir do segundo ínstar (3STT) ou iniciaram a alimentação com tripes nesse ínstar (3SST).

Os testes de alimentação foram realizados em tubos de vidro de $8,5 \mathrm{~cm}$ de comprimento e $2,4 \mathrm{~cm}$ de diâmetro. Em cada tubo era colocada inicialmente uma quantidade de tripes (adultos) suficiente para a alimentação dos predadores por $24 \mathrm{~h}$. Juntamente com os tripes, foi colocado um disco de alface de $2 \mathrm{~cm}$ de diâmetros, assim como uma larva de crisopídeo do ínstar correspondente ao teste realizado. Posteriormente, os tubos foram fechados com PVC laminado.

Foram preparados 7 tubos para cada ínstar do predador. A cada 24 horas fazia-se a contagem do número de tripes consumidos por cada larva. Esta contagem foi obtida a partir do número de sobreviventes subtraído do total fornecido no início de cada período de alimentação.

Utilizou-se delineamento inteiramente casualizado com seis tratamentos (já descritos) e sete repetições.

Os dados foram submetidos à análise de variância, combinada com o teste F. As médias foram comparadas pelo teste de Tukey ao nível de $5 \%$ de probabilidade. Foi feita uma analise gráfica para verificar o consumo médio das larvas ao longo do desenvolvimento. 


\section{RESULTADOS E DISCUSSÃO}

Consumo médio diário - Verificou-se um maior número de Frakliniella schultzei (Trybom) consumido por larva de Chrysoperla externa (Hagen) de 3ํ ínstar, quando comparado com larvas de $1^{\varrho}$ e $2^{\circ}$ ínstar, independentemente dos seus regimes alimentares (Tabela 1).

Entre larvas de $2^{2}$ ínstar não foi constatada a influência da alimentação anterior no consumo de tripes, mostrando-se sempre superior ao número de tripes consumidos por larvas de $1^{0}$ ínstar (Tabela 1).

$\mathrm{O}$ alimento fornecido às larvas, antes da montagem do experimento, influenciou o número de tripes consumidos por larva de 3 ínstar; foi constatado que larvas alimentadas nos ínstares anteriores com Sitotroga cerealella (3SST) apresentaram consumo estatisticamente maior $(19,90)$ do que larvas do mesmo ínstar, que se alimentaram a partir do $2^{\circ}$ ínstar com tripes (3STT) $(12,85)$, e do que as alimentadas desde o $1^{10}$ ínstar com tripes (3TT.T) $(12,74)$ (Tabela 1). Assim larvas provenientes de criação massal, em que a presa alternativa, comumente utilizada, é $S$. cerealella, terão um comportamento melhor do que as que já estiverem presentes nos ínstares anteriores na casa de vegetação, alimentando-se com tripes.

Resultados obtidos por Paine (1992), para larvas de C. carnea, de 3 ínstar, alimentadas com Gynaikotrips ficorum Marchal, demonstraram que o número de tripes consumidos, em 24 horas, foi de 17,91 adultos quando oferecidos 100 indivíduos, sendo próximo ao consumo de $F$. schultzei por larva 3SST.

Período de Desenvolvimento - Verificou-se que larvas de $1^{o}$ ínstar (1T), alimentadas com tripes gastaram em média 5,43 dias para atingir o estágio posterior. Para as de $2^{\circ}$ ínstar, a influência do alimento no período de desenvolvimento de C. externa foi observada; larvas alimentadas no ínstar anterior com ovos de $S$. cerealella (2ST) gastaram um período menor ( 3,14 dias), quando comparadas com as larvas que iniciaram a alimentação com tripes no $1^{\circ}$ ínstar (2TT) (4,00 dias) (Tabela 1). 
Para larvas de $3^{\circ}$ ínstar as avaliações foram realizadas até 15 dias. Nesse intervalo, constatou-se que larvas alimentadas com tripes (3TTT) nos ínstares anteriores não atingiram a fase posterior (pupa). Da mesma forma, larvas que começaram a se alimentar com tripes a partir do $2^{\circ}$ ínstar (3STT), apresentaram mortalidade de $43 \%$, antes de finalizar esse período. Porém larva de $3^{2}$ ínstar alimentadas anteriormente com $S$. cerealella (3SST), levaram em média 9,14 dias para atingir a fase posterior.

Períodos inferiores ao obtidos no presente trabalho, foram demonstrados por Awadallah et al. (1975), ao estudarem C. carnea alimentadas com Trips tabaci Lind. $\left(3,57 ; 2,45 ; 7,46\right.$, para larvas de $1^{2}$, $2^{\circ}$ e $3^{\circ}$ instares, respectivamente) e $G$. ficorum $(3,1 ; 3,0$; e 3,87 para os três ínstares, respectivamente). Essas presas promoveram um ciclo de desenvolvimento curto. Assim, acredita-se serem adequadas. O mesmo não ocorreu no presente trabalho, onde larvas de $C$. externa não finalizaram a fase larval (3TTT e 3STT) ou tiveram um ciclo muito longo (3SST).

\section{Consumo médio para cada ínstar ao longo do desenvolvimento -} Observou-se que, para larvas de $1^{\circ}$ ínstar alimentadas com tripes (1T), o maior consumo ocorreu no período entre 48 e $72 \mathrm{~h}$, após o início da alimentação. Esse consumo foi reduzido a 96 horas, sendo mantido até 120 horas, quando ocorreu a ecdise da maioria das larvas (Figura 1A).

Constatou-se que a variação no número de tripes consumidos entre as larvas 2TT e 2ST foram semelhantes, com maior consumo 48 h após o início do teste, decrescendo perto da ecdise (72 horas) (Figura $1 \mathrm{~B})$.

Verificou-se semelhança no consumo das larvas 3TTT, 3STT e 3SST até 72 horas, quando apresentaram um pico máximo. Posteriormente esse consumo se manteve semelhante entre as larvas 3TTT e 3STT; no entanto, foi sempre inferior ao das larvas 3SST, que, na maioria, empuparam 288 horas após estarem no 3 in ínstar (Figura 1C). 
Tabela 1. Consumo médio diário e período de desenvolvimento médio (dias) de larvas de Chrysoperla extena de $1^{\circ}$ (1T), 20 (2TT e 2ST) e 3o (3TTT, 3STT e 3SST) ínstares, alimentadas com adultos de Frankniella schultzei.

\begin{tabular}{lcc}
\hline Larva & Cosumo médio diário das larvas & Período médio (dias) \\
\hline 1T & $3,32 \mathrm{E}$ & 5,43 \\
2TT & $9,73 \mathrm{D}$ & 4,00 \\
3ST & $10,20 \mathrm{CD}$ & 3,14 \\
3STT & $12,74 \mathrm{BC}$ & 15,00 \\
3SST & $12,85 \mathrm{~B}$ & 12,86 \\
& $19,90 \mathrm{~A}$ & 9,14 \\
\hline
\end{tabular}

Médias seguidas por letras distintas na coluna, diferem entre si pelo teste de Tukey $(\mathrm{P}<0,05)$

\section{CONCLUSÕES}

1. O consumo de F. schultzei, por larvas de C. externa, foi influenciado pelo regime alimentar anterior fornecido ao predador.

2. Larvas de C. externa alimentadas com F. schultzei, tiveram período de desenvolvimento longo ou não concluíram a fase, evidenciando que essas presas foram qualitativamente inadequadas. 


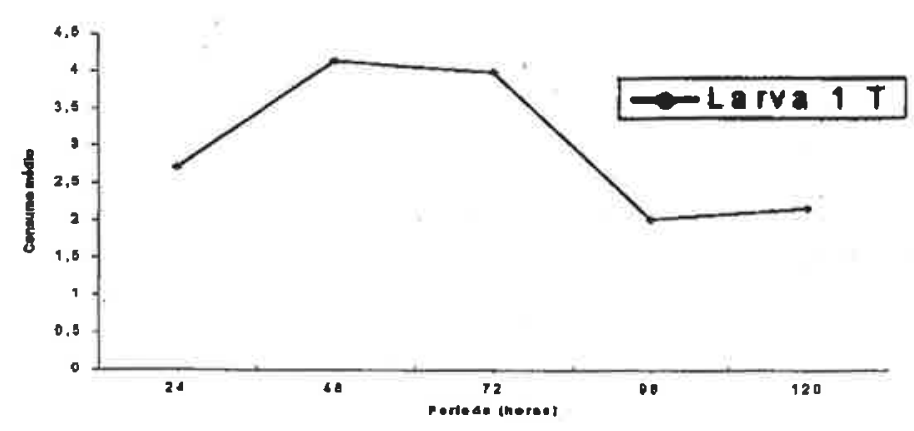

B

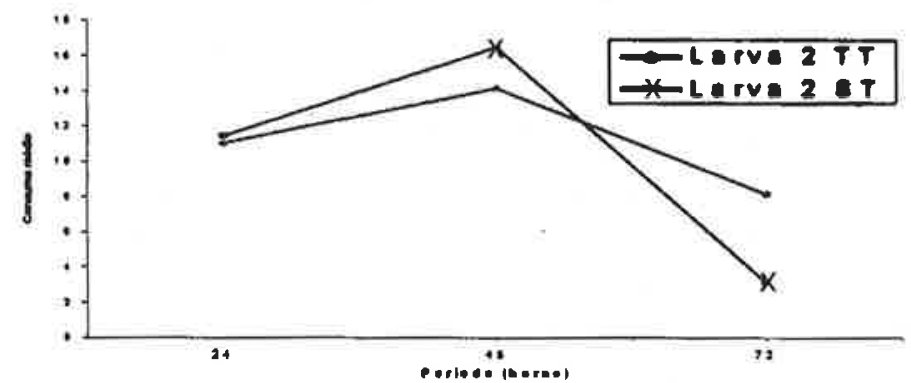

C

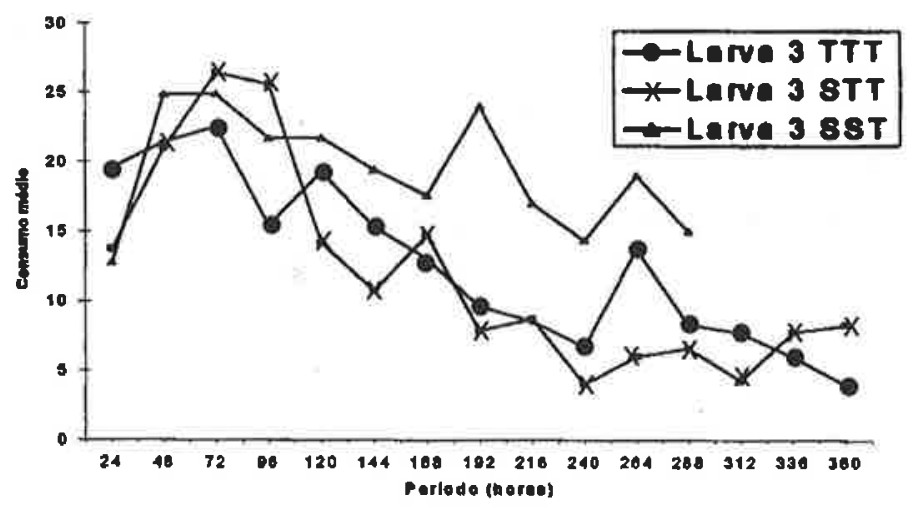

Figura 1. Número de Frankniella schultzei consumidos por larvas de Chrysoperla extena de $1^{\circ}$ (1T) (A), 20 (2TT e 2ST) (B) e $3^{\circ}$ (3TTT, 3STT e 3SST) (C) ínstares, ao longo do desenvolvimento. 


\section{REFERÊNCIAS BIBLIOGRÁFICAS}

ANANTHAKRISHNAN, T. N., 1993. Bionomics of Thrips. Ann. Rev, Entomol., 38:71-92.

AWADALLAH, K. T.; N. A. ABOU-ZEID; M. F. S. TAWFIK., 1975.

Development and Fecundity of Chrysopa carnea Stephens. Bul. Soc. Entomol. d'Egyte, 89:323-329.

FREITAS, S. DE \& O. A. FERNANDES, 1996 Crisopídeos em Agroecossistemas. In: SIMPÓSIO DE CONTROLE BIOLÓGICO, 5, Foz do Iguaçu. Resumos., p. 283.

LEWIS, T., 1973. Thrips: Their Biology, Ecology and Economic Importance. London: Academic Press, 349p.

LIMA, A. C. Ordem Thysanoptera. In: Lima, A.C. Insetos do Brasil. Rio de Janeiro: ENA, 1938, p.405-52.

PAINE, T. D., 1992. Cuban Laurel Thrips (Thysanoptera: Phlaeothripidae) Biology in Southern California: Seasonal Abundance, Temperature Dependent Development, Leaf Suitability, and Predation. Ann. Entomol. Soc. Amer., 85 (2):164-172.

PRINCIPI, M. M., 1984. Neuroptera Chrysopidae and the Possibilities of Their Use in Biological and Integrated Control. Bol. Inst. Entomol. Univer. Studi di Bologna, (38):231-261.

YUDIN, L. S.; B. E. TABASHNIK; J. J. CHO; W. C. MITCHELL., 1988. Colonization of Weeds and Lettuce by Thrips (Thysanoptera: Thripidae). Environ. Entomol.,.17(3):522-526. 